\title{
DC-ELF Characterization of Random Mixtures of Piecewise Nonlinear Media
}

\author{
B. Bianco, ${ }^{1,2 *}$ A. Chiabrera, ${ }^{1,2}$ and S. Giordano ${ }^{2}$ \\ ${ }^{1}$ ICEmB at DIBE, University of Genoa, Genoa, Italy \\ ${ }^{2}$ Department of Biophysical and Electronic Engineering, Genoa, Italy
}

\begin{abstract}
Biological tissues are ensembles of linear and nonlinear, symmetric and asymmetric constituents. As far as their electromagnetic characterization is concerned, they can be modeled as microscopic mixtures of the corresponding material media. Any medium volume can be properly discretized in a finite number of cells which can be modeled as an equivalent three dimensional network of lumped components, in order to characterize its electromagnetic behavior at wavelengths much longer than the relevant average linear size of the constitutive cells. Therefore, any mixture and the corresponding tissue can be characterized in terms of its effective conductance at extremely low frequency, with respect to a reference set of electrodes (ports of the equivalent network). When the above procedure is implemented for evaluating any of the aforesaid conductances, a resulting nonlinear characteristic should be expected. In reality, it may happen that the effect of the constitutive nonlinearities and the related asymmetries are smeared out by the randomness of the interconnections of the lumped components, leading at a macroscopic level to an isotropic constant equivalent conductance, i.e., to an isotropic constant equivalent conductivity of the mixture. The closed form analysis of a random network of nonlinear (piecewise linear) resistors offers a simple but clear cut example of such a property. This result, if extrapolated to biological media, suggests a new hint for explaining why there is no inconsistency between the typical electric characterization of biological tissues as almost linear macroscopic media, by means of their effective conductivity and permittivity, and the nonlinearities of the biochemical processes occurring in the tissue cells. In fact, the nonlinearities may not be observable by means of macroscopic electrical measurements because of the randomized spatial orientation and location of the processes. Bioelectromagnetics 21:145-149, 2000. ๑ 2000 Wiley-Liss, Inc.
\end{abstract}

Key words: dielectric measurement; conductivity; biological tissue; heterogeneous media; nonlinear materials

\section{INTRODUCTION}

The electric characterization of biological tissues in terms of their macroscopic effective conductivities and permittivities vs. frequency, provides an important way to evaluate the spatial average of the electromagnetic (EM) field components inside the tissues as function of the known external sources [Foster and Schwan, 1986; Gabriel et al., 1996]. These local spatial average field components may be used for three purposes. They can provide (i) the Specific Absorption Rate in given tissue volumes for the dosimetric compliance with the current safety standards; (ii) the basis for evaluating possible bioeffects of the EM exposure due to field interactions with some biochemical processes; and (iii) information about the values of some characteristic parameters of the microscopic constituents of the tissue.

From an experimental point of view, the macroscopic behavior of most biological tissues as linear media at the E.M. field intensities typically used for the measurements of their equivalent conductivities and permittivities, is a solid result [Foster and Schwan, 1986; Gabriel et al., 1996] on which the tissue dosimetry is based. Accordingly, the field intensities in situ calculated by computer simulation or measured on phantoms, in order to be compared with the limit set by the standards, consistently imply the linearity of the tissues. On the other hand, biochemistry teaches that most cellular processes are inherently nonlinear [Alberts et al., 1994]. Therefore, if one considers any

Contract grant sponsor: Italian MURST 40\% Program and University of Genoa.

*Correspondence to: Bruno Bianco, ICEmB at DIBE, University of Genoa, Via Opera Pia 11a, 16145 Genoa, Italy.

E-mail: bianco@dibe.unige.it

Received for review 23 February 1999; Final revision received 30 June 1999 
tissue as a true mixture of microscopic constituents, an implicit dogma too often accepted without criticism, is that the tissues's macroscopic linearity is a consequence of the fact that the working point of the microscopic constituents occurs in the linear zone of their characteristics (small signal approximation). Thus, one possible and favorite explanation is that the coupling of biochemical processes to exogenous EM field should be so small that the nonlinearity does not show up in the macroscopic conductivity or permittivity of the tissue. This is true for those dielectric theories whose small signal-linearity assumptions are well justified experimentally. On the other hand, the same argument is sometimes used with the purpose of challenging any possible bioeffects of low intensity EM exposure, below the current safety standards.

We shall prove with a counter example, that in principle the above inference may be sometimes incorrect, because asymmetric nonlinear microscopic constituents of a medium can lead to a macroscopic linear characteristic irrespective of the field intensity, if they are truly randomly oriented. The same conclusion obviously holds if the medium constituents are uniformly distributed in all the directions. The issue can be further clarified considering the example offered by a metal electrode interfacing a fluid. In this case, a nonlinear current-voltage characteristic arises because the contributing transport processes at the metal-fluid interface are strongly correlated with the normal of the geometrical surface. But the situation changes if the surface layer of the metal is porous. In this case the true contact interface becomes very tortuous and randomly oriented, e.g. in porous tantalum and silver and it is no longer coincident with the electrode geometrical surface. The electrode characteristic now becomes (almost) linear.

\section{THE MODEL}

In order to handle the nonlinear problem in a closed form, some simplifying hypotheses must be assumed. We consider a single ideal medium, located in similar regions (cells) made of a nonlinear asymmetric material and exposed to a dc or slowly timevarying electric field. The asymmetric nonlinear regions are randomly oriented, as schematically shown in Figure 1a. For example, with reference to three orthogonal axes $x, y, z$, each region $i$ is characterized by the nonlinear conductivities $g_{x}^{i}, g_{y}^{i}$ and $g_{z}^{i}$. The $X$ axis conductivity $g_{x}^{i}$ can assume at random the value $g_{\mathrm{F}}$ (or $\left.g_{\mathrm{R}}\right)$ if the X-component of the electric field $\mathrm{E}_{x}>0$ and the value $g_{\mathrm{R}}$ (or $g_{\mathrm{F}}$ ) if $\mathrm{E}_{x}<0$, where $g_{\mathrm{F}} \neq g_{\mathrm{R}}$. The same definition applies to $g_{y}^{i}$ and $g_{z}^{i}$.
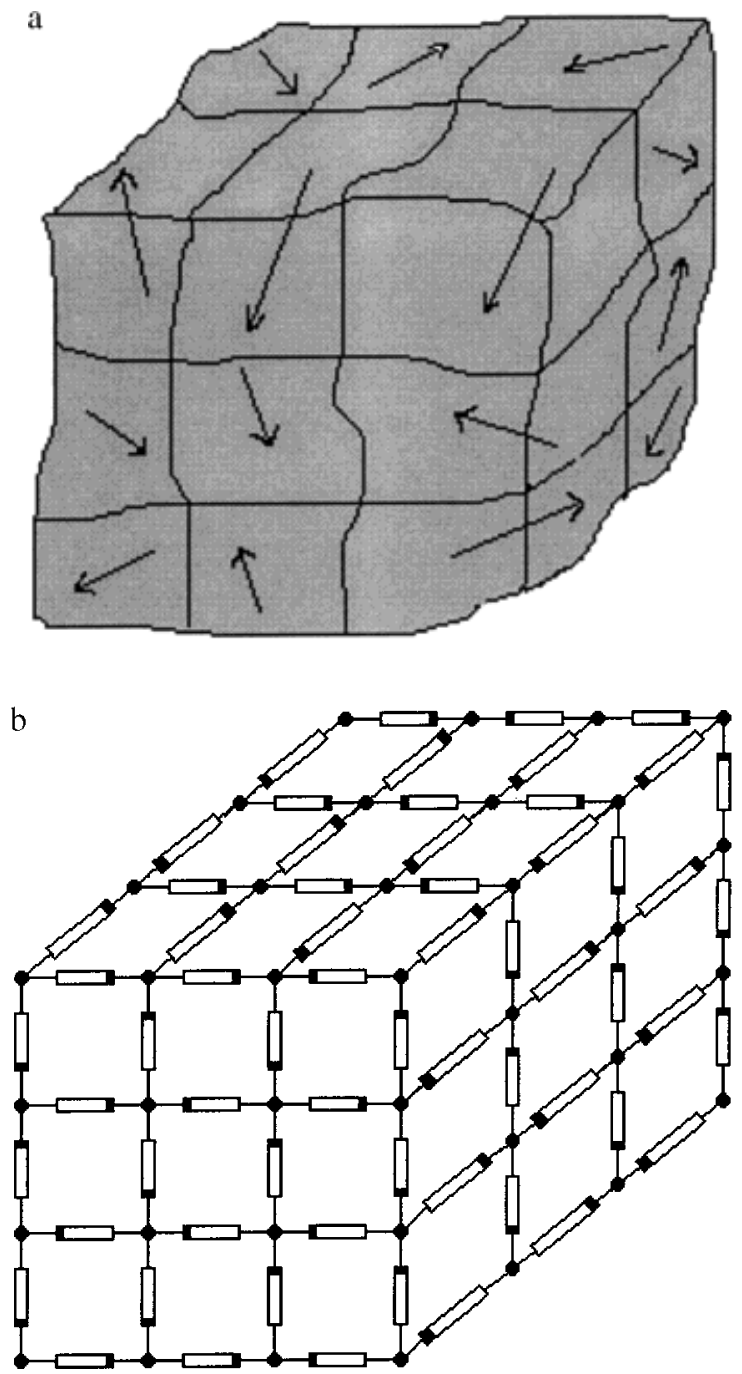

Fig. 1. (a) Schematics of a putative tissue made of regions whose constitutive medium is the same. The electric characteristic of the medium is nonlinear and asymmetric, as schematically indicated by the arrows. The directions are oriented at random. (b) Threedimensional network of identical lumped elements (see Fig. 2), electrically equivalent to the tissue of Figure 1a. We assume, as a first order approximation, that only the interactions with the six closest nodes are important.

It can be safely assumed that, by discretization of the corresponding electromagnetic equation in cartesian coordinates, the resulting mixture can be always modeled by a three dimensional array of nodes, for example arranged in a cubic lattice (Fig. 1b). Each pair of contiguous nodes along the three coordinate axes is randomly interconnected by an asymmetric nonlinear component (one-port).

Let us assume the simple nonlinear (piecewise linear) asymmetric characteristic of each component as shown in Figure 2, which is consistent, for example, with the above conductivities, so that $G_{\mathrm{F}}$ is related to $g_{\mathrm{F}}$ and $G_{\mathrm{R}}$ to $g_{\mathrm{R}}$. The diodes shown in the equivalent 

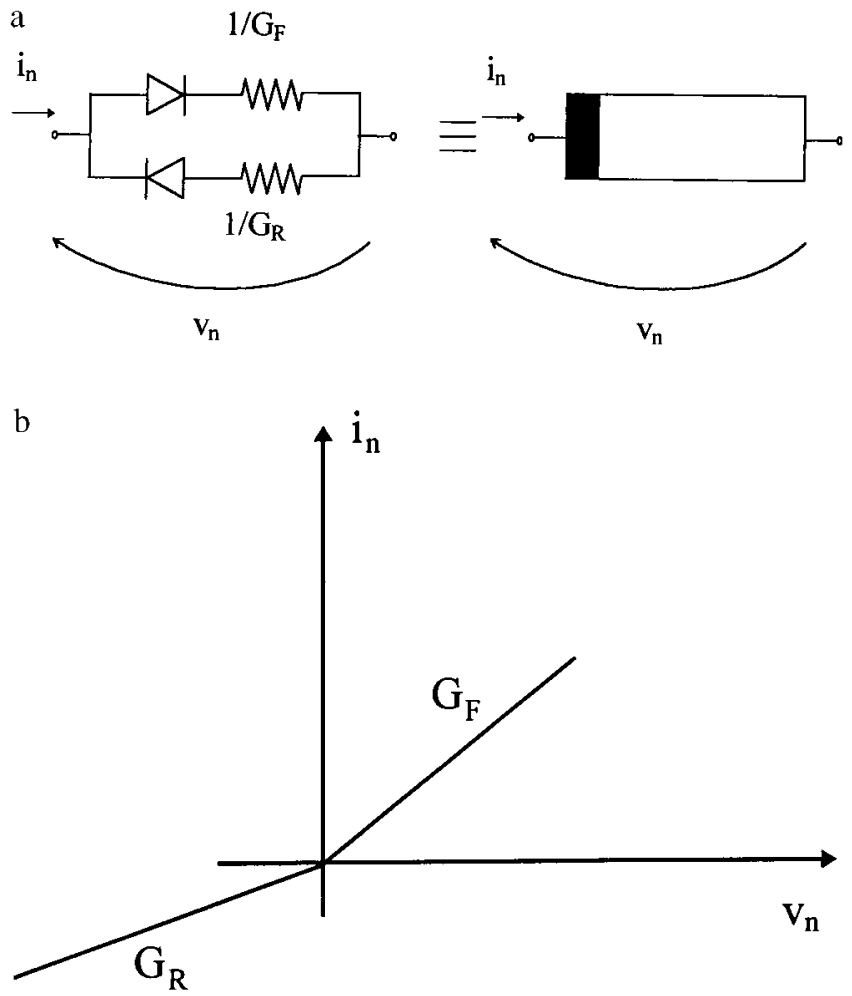

Fig. 2. (a) Schematics of the lumped elements of Figure $1 \mathrm{~b}$. (b) Nonlinear and asymmetric characteristic of the elements of Figure 2a.

circuit of Figure $2 \mathrm{a}$ are ideal, i.e., they act as short circuits if their current is positive and open circuits if their voltage is negative. If $G_{\mathrm{R}}=0$ the characteristic reduces to the model of an ideal diode in series to a resistor $1 / G_{\mathrm{F}}$. The goal is the evaluation of the overall dc "averaged" electrical characteristic conductances related to the network of Figure $1 \mathrm{~b}$, made of the nonlinear asymmetric components of Figure 2, interconnected at random. Any piecewise nonlinear component, being asymmetric, will be oriented in one direction or the other at random, with probability $\frac{1}{2}$.

A one-port can be associated with the network by means of two arbitrary electrodes parallel to the planes of the cubic lattice. Their potential difference is $v$, and their associated current is $i$. It follows that the ensemble average characteristic $\mathrm{f}(v, i)=0$ of any one-port must be symmetric because of the random arrangement of the individual components, so that:

$$
\mathrm{f}(v, i)=\mathrm{f}(-v,-i)=0 .
$$

Given two electrodes which contact a particular instance of the network, one can define a specific value of an applied voltage $v$ and of the related current value $i$ that enters one electrode and leaves the other.
The corresponding sets of the currents $i_{\mathrm{n}}$ and voltages $v_{\mathrm{n}}$ of the n-th component satisfy the Kirchoff laws. The component working point is in the forward $\left(v_{n}>0\right)$ or reverse $\left(v_{\mathrm{n}}<0\right)$ zone of its characteristic.

One can now multiply the current $i_{\mathrm{n}}$ in each device $n$ by the same arbitrary factor $\beta>0$. Then all the voltages $v_{\mathrm{n}}$ are multiplied by $\beta$, without changing the working zone (forward or reverse) of each device. The new sets of values $\beta i_{\mathrm{n}}$ and $\beta v_{\mathrm{n}}$ also satisfy the same Kirchoff laws as before. Thus the overall voltage becomes $\beta v$ and the total current entering and leaving the electrodes is $\beta i$. Therefore, the resulting conductance $G=\beta i /(\beta v)=i / v$ is independent of $\beta$, i.e., of the amplitude level of the applied signal. Therefore $G$ must be constant, irrespective of the individual components being nonlinear. If one lets $v^{\prime}=-v$ and $i^{\prime}=-i$, the same arguments lead to the conclusion that the resulting conductance $G^{\prime}$ is constant and $G^{\prime}=G$ because of the property (l), irrespective of the fact that some individual components may switch to the opposite working zone, with respect to the previous situation. Therefore, since the overall one-port is symmetric, as stated above, it is totally equivalent in average sense to a linear resistor $1 / G$.

The value of $G$ can be easily found in the case of parallel electrodes, both orthogonal to one of the cartesian coordinate axes. To be definite, we consider a cube of $\mathrm{N} \times \mathrm{N} \times \mathrm{N}$ components, oriented at random. For a large $\mathrm{N}$, about half of the components must operate in the forward zone $\left(G_{\mathrm{F}}\right)$ and half in the reverse zone $\left(G_{\mathrm{R}}\right)$, so that the resulting conductance is [Bianco et al., 1982]:

$$
G=\mathrm{N}\left\{G_{\mathrm{F}}+G_{\mathrm{R}}+\left[\left(G_{\mathrm{F}}+G_{\mathrm{R}}\right)^{2}+32 G_{\mathrm{F}} G_{\mathrm{R}}\right]^{1 / 2}\right\} / 8
$$

The computer simulation of the cubic network for $\mathrm{N} \geq 5$ confirms the closed form relationship (2) (see Fig. 3a) in any of its realizations. The result of Figure $3 \mathrm{a}$ proves that the network performs as a linear constant conductance $G=13.03$ [a.u.], i.e., $i(\mathrm{t})=(13.03) v(\mathrm{t})$ for slowly time-varying external signals. However, the external signals may be nonlinearly coupled to the voltage $v_{m}$ and the current $i_{m}$ of any individual component, labeled with the suffix $m$. For example, pure sinusoidal external signals $v$ and $i$ can induce a non sinusoidal current in the $\mathrm{m}$-th element, as shown in the example of Figure $3 \mathrm{~b}$.

Therefore the time average $\left\langle i_{m}\right\rangle$ is different from zero, while the time averages $\langle i\rangle$ and $\langle v\rangle$ are exactly zero. This implies a constant flux of current carriers in the m-th branch. If such a situation is extrapolated 


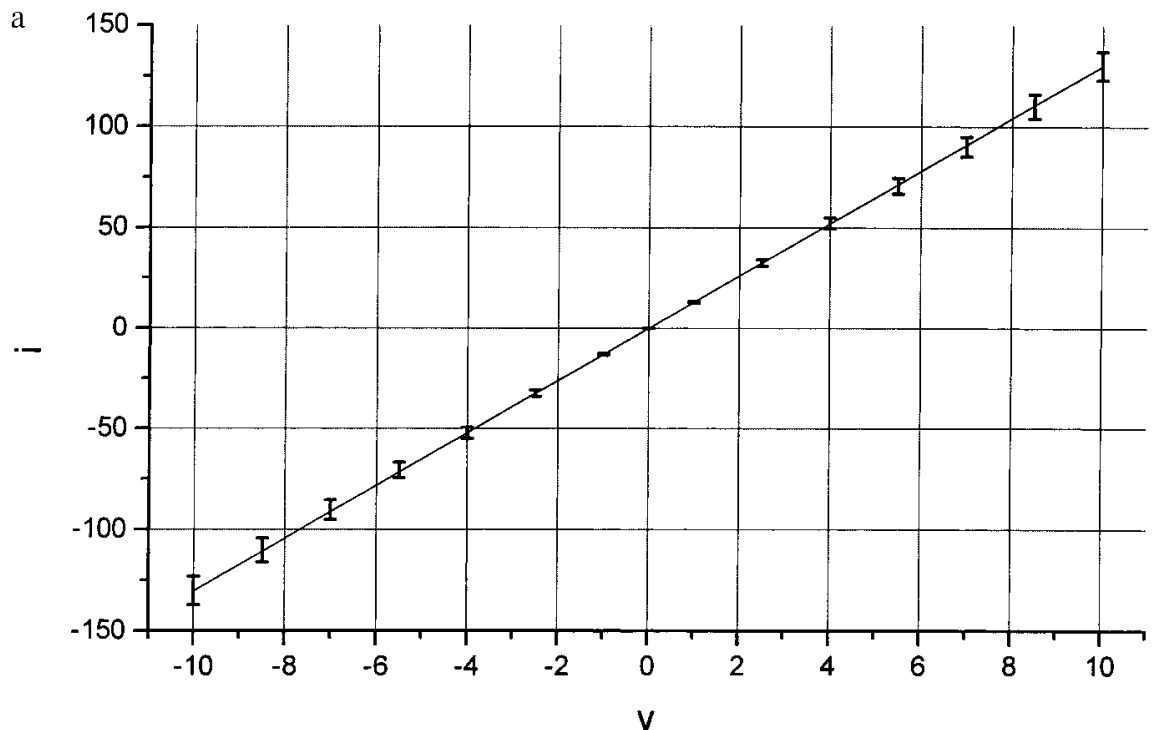

b
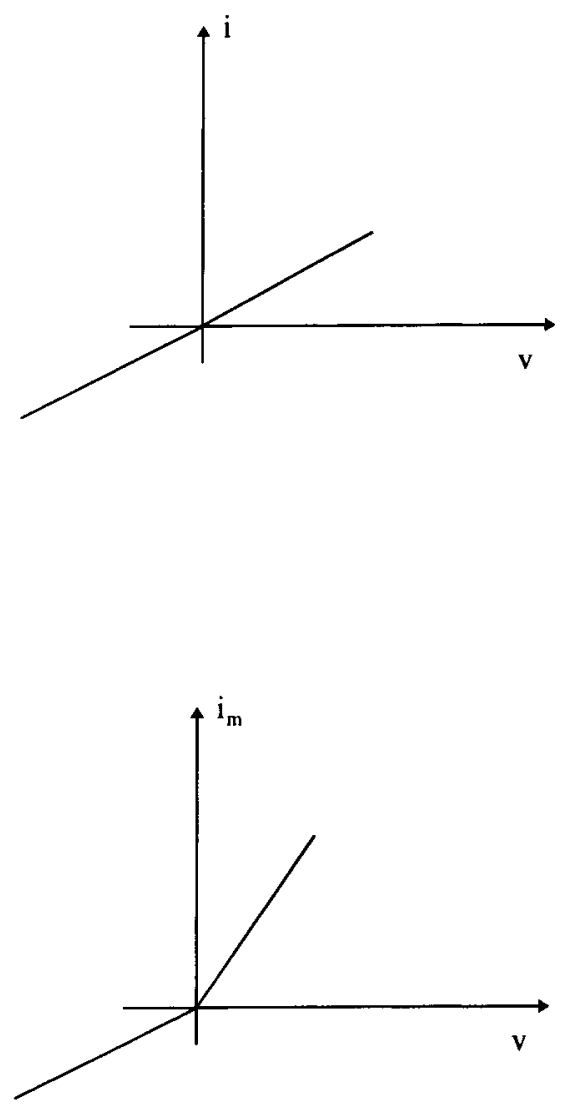

V

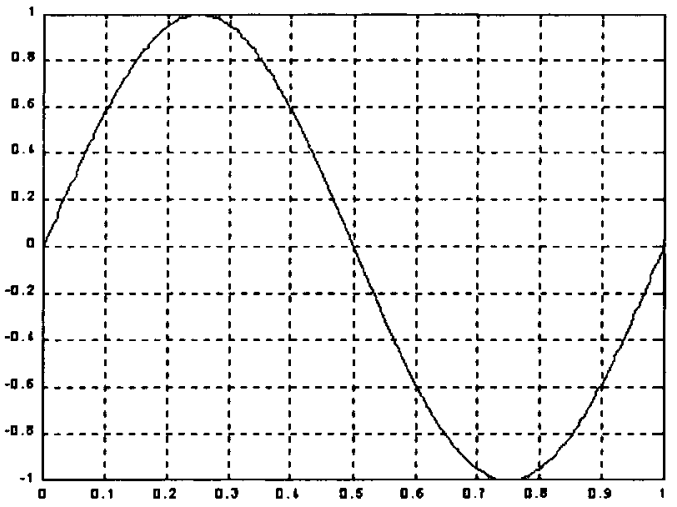

$i_{m}$

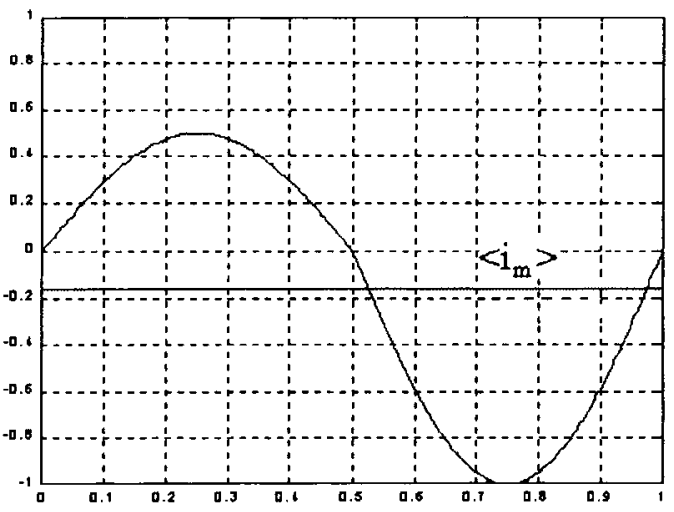

$\mathrm{t} / \mathrm{T}$

Fig. 3. (a) Example of computer simulation (performed using the nonlinear relaxation method) of 50 different realizations of the network of Figure $1 \mathrm{~b}$ with $\mathrm{N}=6, G_{\mathrm{F}}=1$ [a.u.], $G_{\mathrm{R}}=4$ [a.u.]. (b) If a sinusoidal signal $v=\sin (2 \pi \mathrm{t} / \mathrm{T})$ is applied to any of the realizations of the network of Figure $3 \mathrm{a}$, the current is $i=(13.03) \sin (2 \pi \mathrm{t} / \mathrm{T})$, since the network behavior is linear. However, the corresponding voltage $v_{m}$ and current $i_{m}$ of one individual component $m$ of a specific realization of the network are not sinusoidal and rectification occurs (dashed line $\left\langle i_{m}\right\rangle$ ), because of the intrinsic nonlinear characteristic (Fig. 2b) of the component. 
to an EM exposed biological system, a constant ionic flux may elicit a biological response, if exposure is protracted in time. One could argue that high endogenous fields, large ionic gradients, and noise (not considered in this paper) should overwhelm $\left\langle i_{m}\right\rangle$. This is not the case because, when the exposure is set equal to zero, these endogenous forces always balance to give a value of $\left\langle i_{m}\right\rangle$ exactly equal to zero, irrespective of their intensity.

An analogous result holds if the following characteristic is used: $i_{n}=I_{s}\left(\exp \left(\alpha v_{n}-1\right)\right.$ where $I_{s}$ and $\alpha$ are two constants [Bianco, 1998]. The proof is rather cumbersome, so that the characteristic shown in Figure $2 \mathrm{~b}$ has been chosen in order to better communicate with a wider audience.

A similar result can obviously be obtained in terms of the equivalent conductivity of the mixture. If the average size of the constitutive asymmetric regions of Figure $1 \mathrm{a}$ is $\mathrm{L}$ and each region is characterized at random by the two asymmetric values $g_{\mathrm{F}}$ and $g_{\mathrm{R}}$ (respectively for the forward and reverse characteristic) of its conductivity in a given direction, the related conductances are $G_{\mathrm{F}}=\mathrm{L} g_{\mathrm{F}}$ and $G_{\mathrm{R}}=\mathrm{L} g_{\mathrm{R}}$. The proportionality constant is the same. From a macroscopic point of view, if $g$ is the overall effective conductance of the mixture, $G=\mathrm{NL} g$ because the size of the overall cube is NL. Therefore, from Eq. (2) it follows in straightforward way the relationship:

$$
g=\left\{g_{\mathrm{F}}+g_{\mathrm{R}}+\left[\left(g_{\mathrm{F}}+g_{\mathrm{R}}\right)^{2} /+32 g_{\mathrm{F}} g_{\mathrm{R}}\right]^{1 / 2}\right\} / 8
$$

which relates $g_{\mathrm{F}}$ and $g_{\mathrm{R}}$ to the equivalent conductivity of the mixture. Also, the resulting equivalent conductivity $g$ of the mixture does not depend on the intensities of the current density which flows in each constitutive region and of the local electric field.

\section{CONCLUSIONS}

We conclude that a putative medium, made of randomly oriented nonlinear asymmetric regions and modeled by a cubic network of nonlinear (piecewise linear) elements, i.e., ideal diodes with each one being in series to a resistor, is characterized by a DC-ELF conductivity which is equivalent to a linear medium of constant conductivity. The intrinsic asymmetry and nonlinearity of each component are not observable at macroscopic level, because they are smeared out by the randomness of the interconnections. Such a rather rigorous result offers a new perspective if extrapolated to some aspects concerning the coupling between EM exposure and cellular biochemical processes in biological media. As shown in the example of Figure $3 \mathrm{~b}$, the fact that the electrical behavior of a biological tissue is equivalent to an almost linear medium at a given field intensity is not inconsistent with the possibility that the same field can effectively couple to the intrinsic nonlinearities of the biochemical constituents of the tissue. Therefore the same EM exposure, if protracted in time, could elicit unpredictable biological responses.

\section{ACKNOWLEDGMENT}

The authors acknowledge the useful criticisms of the reviewers.

\section{REFERENCES}

Alberts B, Bray D, Lewis J, Raff M, Roberts K, Watson JD. 1994. Molecular biology of the cell. New York: Garland.

Bianco B, Degani G, Parodi M. 1982. A circuit theory approach for deriving the electrical properties of mixtures. Proc. of the 1982 International IEEE Symposium on Circuits and Systems, Rome.

Bianco B. 1998. Macroscopic linear electric characteristic of a random network of diodes, ICEmB Internal Report 5/1998, University of Genoa, Italy.

Foster KR, Schwan HP. 1986. Dielectric permittivity and electrical conductivity of biological materials. In: Polk G, Postow E, editors. CRC Handbook of Biological Effects of Electromagnetic Fields. Boca Raton: CRC Press. p 27-98.

Gabriel C, Gabriel S, Courthomt E. 1996. The dielectric properties of biological tissues. 1. Literature survey. Physics in Medicine and Biology 41:2231-2249. 